\title{
Electronic health record implementation: conversion can be hard to swallow
}

\section{Introduction}

Health care organizations are constantly evolving and becoming more complex. The electronic medical record (EHR) is a prime example of this evolution and complexity. It has demonstrated efficacy in patient care, reduction in errors, lower health care costs, and improved provider and patient satisfaction. ${ }^{1}$ However, the implementation of the EHR platform and conversions of one EHR to another can prove to have challenges that were not predicted. Diligence is required to avoid unintended consequences that may arise during EHR implementation or conversion. The quality and safety of patient care rely on seamless integration of clinical practices, integration of information, and the integrity and reliability of the EHR system. ${ }^{2}$ When quality of care is compromised quick action, cause determination, and interventions are required to avoid risks to patient care.

\section{Problem identification}

An example of this can be demonstrated through a case evaluation. During a recent EHR conversion, a small increase in patients diagnosed with aspiration pneumonia was noted. Hospital protocol requires all patients diagnosed with community-acquired pneumonia be screened for dysphagia, difficulty swallowing, a known risk factor for aspiration pneumonia. ${ }^{3}$ On further examination, screening had not been documented on randomly selected audited patients. The rate of aspiration pneumonia readmissions for community-acquired pneumonia (CAP) had previously been consistent, an increase in readmissions for this diagnosis group was alarming and quickly created the need for further investigation. Since the timing of the increase in readmissions coincided with a new EHR implementation, a system analysis was needed. During implementation, an EHR system can be fragmented and requires navigation skills to avoid errors. ${ }^{1}$ Due to the organization's aims for high reliability, a root-cause analysis was implemented to analyze a potential system cause. A root-cause analysis is a process that was once used to identify the underlying cause for failure or error, but it is now used frequently to identify the cause of contributing factors and event clusters as a proactive tool. ${ }^{4}$

\section{Root cause analysis}

Utilizing a proactive root-cause analysis allowed for examination at multiple levels. The team processed potential errors that could occur due to multiple failures and not isolated individual errors (AHRQ, 2016). The team focused on defining the problem, finding solutions, and strategy implementation. The importance of quality documentation requires clinical knowledge in developing nursing documentation and attention to detail, especially during EHR building and the transition process.

In the root-cause analysis, two types of errors were identified: active errors and latent errors. Active errors are related to the person using the system, or the interface and latent errors are related to failures in the system design. ${ }^{5}$ The care of patients that were readmitted with aspiration pneumonia was reviewed utilizing a systems process, including reviewing pneumonia orders and protocols from the previous admission. In this case scenario, the root analysis discovered both types of errors. Several latent errors were noted. First, the new EHR system had multiple possible locations for the dysphagia screen

\author{
Volume 5 Issue 2 - 2018 \\ LaDawna Goering \\ Department of Nursing, Northern Illinois University, USA
}

Correspondence: LaDawna Goering, Department of Nursing, Northern Illinois University, USA,

Email ladawnagoering@me.com

Received: January 07, 2018| Published: March 07, 2018

documentation and order. Secondly, the primary location for the dysphagia screen had moved to a different location from the previous EHR system, which may have led to a process error. Thirdly, the daily assessment for dysphagia screen was in a daily care activities list and not in a physical assessment area. The screening was previously located in a primary physical assessment area. This emphasizes the importance of clinical expertise in EHR documentation creation and implementation practice.

In a separate process break down it was noted that although all patients with pneumonia are required to have a dysphagia screening or nurse bedside swallow screen per hospital protocol, the pneumonia protocol order set was required to prompt the order for the screen and not all patients with pneumonia had protocol utilization. Two additional latent errors were also noted in the admission screening. The admission screening, which includes a required swallow evaluation, can be bypassed, and the screening instructions were not linked to staff use. Positive findings on the screen were to generate a nutrition and dietary services consult without speech therapy notification, this addressed secondary systems errors.

\section{Plan development}

From the root-cause analysis, an action plan was designed. First, the information technologist specialists met with an interdisciplinary clinical team to design changes. The admission screening and dysphagia screening tools were adjusted to add the hospital screening guideline instructions for nursing staff. Second, a positive dysphagia screening on admission was changed to generate both speech and nutrition therapy referrals. Third, a best practice alert was added for all patients with a pneumonia diagnosis to remind all providers that a dysphagia screen should be completed for this at-risk population group; this allowed capture for those patients who were not under a pneumonia protocol utilization. And a dysphagia screen link was added to the daily assessment under nursing care. This systems approach should improve safety and pneumonia quality care while addressing both active error and EHR systems design errors.

\section{Plan implementation}

The EHR system changes required a dedicated interdisciplinary team to develop a specific interventional plan. The staff required 
procedure and EHR documentation education regarding the change implementation. This opportunity was utilized to reinforce pneumonia dysphagia screening requirements for pneumonia, as well as dysphagia screening techniques. Information technology reviewed EHR documentation, dysphagia screening features, and assisted with documentation changes and questions.

\section{Plan evaluation}

Random audits were conducted for dysphagia screening completion and aspiration pneumonia readmissions were monitored for continued reliability. The importance of team integration for organization-wide intervention led to the successful implementation of this strategy to identify potential aspiration pneumonia patients and improved pneumonia quality of care. This required an interdisciplinary team effort of information specialists, nurses, health data analysts, and speech therapists. Continual EHR design structure and improvements are being made through root cause analysis, communication, and feedback.

\section{Lessons learned}

Effective EHR implementation and conversions require integrated teams of clinicians and information technology specialists. Root cause analysis in healthcare data systems can be an effective proactive tool to improve quality care, preventing risk, and promote best practice.
Ultimately, the continual identification of system vulnerabilities or deficiencies with prompt evaluation and team collaboration for improvements is the best defense against errors.

\section{Acknowledgements}

None.

\section{Conflict of interest}

The author declares that there is no conflict of interest.

\section{References}

1. Han H, Lopp L. Writing and reading EHR documentation: An entirely new world. Med Educ Online. 2013;18:1-7.

2. Bowman S. Impact of electronic health record systems on information integrity: Quality and safety implications. Perspect Health Inf Manag. 2013;10(1):1-18

3. Atman K, Yu G, Schaefer SD. Consequences of dysphagia in the hospitalized patient: Impact on prognosis and hospital resources. Arch Otolaryngol Head Neck Surg. 2010;136(8):784-789.

4. Joint Commission. Root cause analysis in health care: Tools and techniques. Joint Commission Resources. 5th edn. Oak Brook, IL. 2015.

5. Agency for Healthcare Research and Quality. Root cause analysis. Patient Safety Network. 2016. University of California, San Francisco, USA. 